\title{
Use of culture and molecular analysis to determine the effect of antibiotic treatment on microbial community diversity and abundance during exacerbation in patients with cystic fibrosis
}

\author{
M M Tunney, ${ }^{1}$ E R Klem, ${ }^{2}$ A A Fodor, ${ }^{3}$ D F Gilpin, ${ }^{1}$ T F Moriarty, ${ }^{1}$ S J McGrath, \\ M S Muhlebach, ${ }^{4}$ R C Boucher, ${ }^{2,5}$ C Cardwell, ${ }^{6}$ G Doering, ${ }^{7} \mathrm{~J} S$ Elborn, ${ }^{6}$ \\ M C Wolfgang ${ }^{2,8}$
}

- Additional data are published online only. To view these files please visit the journal online (http://thorax.bmi.com).

'School of Pharmacy, Queen's University Belfast, UK ${ }^{2}$ Cystic Fibrosis/Pulmonary Research and Treatment Center, University of North Carolina at Chapel Hill, Chapel Hill, North Carolina, USA

${ }^{3}$ Department of Bioinformatics and Genomics, University of North Carolina at Charlotte, Charlotte, North Carolina, USA ${ }^{4}$ Department of Paediatrics, University of North Carolina at Chapel, Chapel Hill, North

Carolina, USA

${ }^{5}$ Department of Medicine, University of North Carolina at Chapel Hill, Chapel Hill, North Carolina, USA

${ }^{6}$ Centre for Infection and Immunity, School of Medicine, Dentistry and Biomedical Sciences, Queen's University Belfast, UK

${ }^{7}$ Eberhard-Karls-Universtät, Hygiene-Institut, Department of General and Environmental Hygiene, Wilhelmstrasse, Tübingen, Germany ${ }^{8}$ Department of Microbiology and Immunology, University of North Carolina at Chapel Hill, Chapel Hill, North Carolina, USA

\section{Correspondence to}

Michael M Tunney, School of

Pharmacy, Queen's University Belfast, 97 Lisburn Road, Belfast BT9 7BL, UK;

m.tunney@qub.ac.uk

MMT, JSE and MCW contributed equally to this study.

Received 19 February 2010 Accepted 16 December 2010 Published Online First 26 January 2011

\section{ABSTRACT}

Background Anaerobic bacteria are increasingly regarded as important in cystic fibrosis (CF) pulmonary infection. The aim of this study was to determine the effect of antibiotic treatment on aerobic and anaerobic microbial community diversity and abundance during exacerbations in patients with CF.

Methods Sputum was collected at the start and completion of antibiotic treatment of exacerbations and when clinically stable. Bacteria were quantified and identified following culture, and community composition was also examined using culture-independent methods. Results Pseudomonas aeruginosa or Burkholderia cepacia complex were detected by culture in $24 / 26$ samples at the start of treatment, 22/26 samples at completion of treatment and 11/13 stable samples. Anaerobic bacteria were detected in all start of treatment and stable samples and in 23/26 completion of treatment samples. Molecular analysis showed greater bacterial diversity within sputum samples than was detected by culture; there was reasonably good agreement between the methods for the presence or absence of aerobic bacteria such as $P$ aeruginosa $(\kappa=0.74)$ and $B$ cepacia complex $(\kappa=0.92)$, but agreement was poorer for anaerobes. Both methods showed that the composition of the bacterial community varied between patients but remained relatively stable in most individuals despite treatment. Bacterial abundance decreased transiently following treatment, with this effect more evident for aerobes (median decrease in total viable count $2.3 \times 10^{7} \mathrm{cfu} / \mathrm{g}, \mathrm{p}=0.005$ ) than for anaerobes (median decrease in total viable count $3 \times 10^{6} \mathrm{cfu} / \mathrm{g}, \mathrm{p}=0.046$ ).

Conclusion Antibiotic treatment targeted against aerobes had a minimal effect on abundance of anaerobes and community composition, with both culture and molecular detection methods required for comprehensive characterisation of the microbial community in the CF lung. Further studies are required to determine the clinical significance of and optimal treatment for these newly identified bacteria.

Chronic bacterial pulmonary infection with recurrent infective exacerbations results in an irreversible decline in lung function in patients with cystic fibrosis (CF) and early death. ${ }^{1}$ Routine bacterial culture methods identify the aerobic bacteria Pseudomonas aeruginosa, Staphylococcus aureus and
Burkholderia cepacia complex ${ }^{2}$ as the principal pathogenic bacteria in adult CF pulmonary infection.

The observation that anaerobic conditions exist in the lungs of patients with $\mathrm{CF}^{3}$ has prompted the search for infection in CF mucus by obligate anaerobes. Studies using molecular detection by PCR and terminal restriction fragment length polymorphism analysis (T-RFLP) suggest that anaerobes are present in CF sputum and are part of a highly diverse and metabolically active polymicrobial community. ${ }^{4-7}$ In a recent study we used strict anaerobic bacteriological culture techniques and detected a spectrum of potentially pathogenic anaerobic species including Prevotella, Veillonella and Actinomyces in high numbers (up to $9 \times 10^{7} \mathrm{cfu} / \mathrm{g}$ sputum) from $42 / 66$ (64\%) sputum samples collected from patients with clinically stable CF. $^{8}$ Subsequent culture ${ }^{9} 10$ and molecularbased $^{9} 11{ }^{12}$ studies have also detected anaerobes in lower airway secretions from adults and children with stable CF, indicating that anaerobes may contribute at all stages of CF lung disease. Anaerobic bacteria are therefore potentially significant pathogens in CF lower respiratory tract infection and their role in disease progression may have been underestimated. ${ }^{8} 11$

If anaerobic bacteria are active and contribute to infection and inflammation in the CF lung, their presence in high numbers may require a change in the antibiotics used for treatment of pulmonary exacerbations to ensure an optimal outcome for the patient. In this study we assessed both aerobic and anaerobic bacterial community composition at initiation and completion of standard antibiotic treatment of an acute exacerbation. Furthermore, to evaluate the effect of antimicrobial therapy on these diverse organisms, we studied longitudinal changes in bacterial load and diversity during exacerbation and when clinically stable. Given that the majority of bacterial species are refractory to culture, ${ }^{13}$ we also compared the use of both aerobic and anaerobic culture and culture-independent molecular techniques for determining microbial community composition.

\section{METHODS \\ Patient selection and collection of samples}

During a 12-month period (February 2006 to January 2007), patients who had a pulmonary 
exacerbation in the previous year were recruited to the study either during an outpatient visit if they were clinically stable or on admission to the hospital for antibiotic treatment of an exacerbation. Subsequent samples were collected when these patients presented with an exacerbation or at their next routine outpatient visit, respectively. 'Clinically stable' was defined as no change in symptoms, forced expiratory volume in $1 \mathrm{~s}\left(\mathrm{FEV}_{1}\right)$ within $10 \%$ of best value in the previous 6 months and no new antibiotics started. Exacerbations were defined according to the criteria of Fuchs et al. ${ }^{14}$ For patients with an exacerbation, samples were collected at initiation of antibiotics ( $24 \mathrm{~h}$ before to a maximum of $48 \mathrm{~h}$ after the first dose of intravenous antibiotics) and at completion of antibiotic treatment ( $24 \mathrm{~h}$ before to a maximum of $48 \mathrm{~h}$ after last dose of intravenous antibiotics).

Spontaneously expectorated sputum was collected and transported within $15 \mathrm{~min}$ to the laboratory for processing in an anaerobic cabinet. All samples were divided with one aliquot immediately processed for culture and the second aliquot frozen and stored at $-80^{\circ} \mathrm{C}$ for subsequent molecular detection at the University of North Carolina at Chapel Hill.

\section{Bacterial culture detection}

Culture and subsequent detection of isolates in sputum samples was performed as previously described ${ }^{8}$ with all bacteria detected quantified by total viable count (TVC) and identified by PCR and sequencing of $16 \mathrm{~S}$ ribosomal genes, a frequently used alternative to phenotypic methods of bacterial identification. $^{15}$

\section{Molecular detection}

Detailed procedures for molecular-based bacterial detection are provided in the online supplement. Briefly, DNA was extracted from frozen $\left(-80^{\circ} \mathrm{C}\right)$ sputum samples and subjected to both quantitative real-time PCR (qPCR) analysis to determine total bacterial load and terminal restriction fragment length polymorphism analysis (T-RFLP) to assess bacterial diversity and for classification of bacterial taxa. ${ }^{16}{ }^{17}$ For qPCR, samples were analysed in duplicate at three different concentrations using a previously published ${ }^{16}$ set of universal primers and TaqMan probe that amplify a conserved fragment of the bacterial $16 \mathrm{~S}$ ribosomal RNA (rRNA) gene. For T-RFLP analysis, the $16 \mathrm{~S}$ rRNA gene was PCR amplified using Bac8f and Bac926r primers fluorescently labelled at the $5^{\prime}$ end. The resulting PCR products were digested in two separate reactions with the restriction endonucleases HaeIII and HhaI and the length of labelled terminal fragments was determined by capillary electrophoresis.

\section{Patient characteristics}

For each patient, sex, age, weight, lung function $\left(\mathrm{FEV}_{1}\right)$ and serum inflammatory markers ( $C$ reactive protein (CRP), white cell count (WCC)) were obtained at each time point. Information on intravenous antibiotics for treatment of an acute exacerbation was also collected.

\section{Statistical analysis}

A detailed description of the statistical methods is provided in the online supplement. Briefly, participant characteristics before and after antibiotic treatment were compared using paired samples $t$ test or Wilcoxon signed rank tests as appropriate. Kappa statistics were calculated to measure agreement between the presence or absence of bacteria detected by culture and T-RLFP. Viable counts were compared at each time point and before and after antibiotic treatment using Wilcoxon signed rank tests. Comparison between three time points simultaneously were made using the Friedman test. The $\log _{10}$ qPCR was compared before and after treatment using a paired samples t test.

\section{RESULTS}

\section{Patient characteristics and sputum samples}

Twenty-three patients were enrolled and sputum samples were collected at initiation and completion of intravenous antibiotic treatment. The mean age was 24.9 years (range 18-41). Three of the patients were treated twice for an exacerbation during the course of the study resulting in a total of 26 matched pairs of initiation/completion of treatment samples. Sputum was also collected from 13 of these patients (mean age 27.0 years (range 19-41)) when clinically stable, with seven samples collected before exacerbation, five samples collected after exacerbation and one sample collected between treatment of two separate exacerbations. The mean time interval between collection of stable and exacerbation sputum samples was 155 days (range 19-281) and 137 days (range 15-231) for samples collected after exacerbation.

Patient characteristics are presented in table 1. The mean duration of intravenous antibiotic therapy was 13 days (range 7-24), with all patients improving clinically. Lung function $\left(\mathrm{FEV}_{1}\right)$ increased significantly by an average of $0.4 \mathrm{l}$ and CRP and WCC decreased significantly following antibiotic treatment of an exacerbation by medians of $7.6 \mu \mathrm{g} / \mathrm{dl}$ and $2.5 \times 10^{9} / 1$, respectively (table 1). Owing to the wide range of antibiotic regimens used to treat exacerbations (see table 1 in online supplement) and the small sample size, we were unable to determine if there was a relationship between use of specific antibiotics and change in anaerobic bacterial load following treatment. Patients primarily received oral azithromycin or inhaled colistin or tobramycin as chronic maintenance treatment. Similarly, owing to the small sample size, we were unable to determine if there was any correlation between antibiotic prophylaxis and presence/absence of any particular bacterial species.

\section{Bacterial culture and identification of isolates}

The predominant aerobic pathogens were present in high abundance (up to $5 \times 10^{8} \mathrm{cfu} / \mathrm{g}$ sputum) in $24 / 26$ samples at the start of treatment (P aeruginosa, 16; B cepacia complex, 8), 22/26 at completion of treatment $(P$ aeruginosa, $15 ; B$ cepacia complex, 7) and 11/13 stable samples (P aeruginosa, 9; $B$ cepacia complex, 2) (see figure 1a in online supplement and table 2). Only one patient (patient 19), who experienced two exacerbations during the study period, had neither $P$ aeruginosa nor $B$ cepacia complex cultured in the sputum at all five time points. Furthermore, $P$ aeruginosa was cultured from one patient (patient 7) when stable and at initiation of antibiotic treatment but not at completion of treatment. Similarly, B cepacia complex was cultured from one patient (patient 3 ) at initiation but not at completion of antibiotic treatment. With these exceptions, the patients had the same aerobic bacteria in sequential sputum samples (see figure $1 \mathrm{a}$ and table 3 in online supplement).

Anaerobic bacteria were detected in high numbers (up to $3 \times 10^{7} \mathrm{cfu} / \mathrm{g}$ sputum) in samples from all patients prior to commencing antibiotic therapy with up to five different species detected per sputum sample (see figure 1a in online supplement). Of the anaerobes detected, Veillonella species (14 samples) and Prevotella species (6 samples) were predominant. At completion of antibiotic treatment, anaerobic bacteria were detected by culture in 23/26 samples, with Veillonella (14 samples) and Prevotella (7 samples) again most prevalent. Anaerobic bacteria 
Table 1 Patient characteristics at the start and completion of antibiotic treatment for an exacerbation and when stable

\begin{tabular}{|c|c|c|c|c|c|c|c|}
\hline & $\begin{array}{l}\text { Start of IV } \\
\text { antibiotics } \\
\text { Mean (SD) } \\
(n=26)\end{array}$ & \multicolumn{3}{|c|}{ Completion of IV antibiotics } & \multicolumn{3}{|l|}{ Stable } \\
\hline Weight (kg) & $59.25(10.70)$ & $59.09(10.93)$ & $-0.16(-0.48$ to 0.17$)$ & 0.33 & $59.27(12.92)$ & $+0.28(-0.05$ to 0.60$)$ & 0.09 \\
\hline $\mathrm{FEV}_{1}(\mathrm{I})$ & $1.65(0.76)$ & $2.05(0.84)$ & $+0.39(0.21$ to 0.58$)$ & $<0.001$ & $1.71(0.66)$ & $+0.12(-0.05$ to 0.30$)$ & 0.16 \\
\hline $\operatorname{CRP}\left(\mathrm{mg} / \mathrm{ll}^{*}\right.$ & $11.7(3.8-28.8)$ & $7.4(3.5-14.5)$ & $-7.6(-2.0$ to -21.5$)$ & 0.005 & $13.0(2.8-33.5)$ & $+0.5(-33.5$ to 15.8$)$ & 0.91 \\
\hline WCC $\left(\times 10^{9} /\right)^{*}$ & $10.1(8.1-14.2)$ & $7.2(6.0-10.3)$ & $-2.5(-4.7$ to -0.8$)$ & 0.01 & $12.5(10.2-16.1)$ & $+1.9(-3.7$ to +4.5$)$ & 0.58 \\
\hline
\end{tabular}

*As CRP and WCC are skewed, the median and IOR are presented along with the median difference from start $(95 \% \mathrm{Cl})$ and the $\mathrm{p}$ value from the Wilcoxon signed rank test.

CRP, C reactive protein; FEV ${ }_{1}$, forced expiratory volume in $1 \mathrm{~s}$; IV, intravenous; WCC, white cell count.

were also detected in all 13 samples from stable patients, with Veillonella (2 samples), Actinomyces (3 samples) and Prevotella (2 samples) species being the most common. Streptococci, which grew under either aerobic or anaerobic conditions, were detected by culture in 12 and 7 initiation and completion of treatment samples, respectively. Overall, culture results showed that, although the composition of the bacterial community varied between patients, it remained relatively stable within the majority of individuals despite antibiotic treatment, with similar numbers and genera of aerobic and anaerobic species detected at each sampling time point (see figure 1 and online table 3 ).

Table 2 Bacteria detected by culture and molecular analysis (terminal restriction fragment length polymorphism, T-RFLP) in sputum samples $(n=64)$ collected from adult patients with cystic fibrosis

\begin{tabular}{|c|c|c|}
\hline \multirow[b]{2}{*}{ Genera } & \multicolumn{2}{|c|}{$\begin{array}{l}\text { Number of samples in which } \\
\text { genera detected by: }\end{array}$} \\
\hline & Culture & T-RFLP* \\
\hline Achromobacter & 1 & 3 \\
\hline Actinomyces & 23 & 3 \\
\hline Atopobium & 6 & 1 \\
\hline Bacillus $†$ & 2 & ND \\
\hline Burkholderia & 17 & 16 \\
\hline Corynebacterium & 1 & 1 \\
\hline Devosia & 0 & 2 \\
\hline Finegoldia† & 1 & ND \\
\hline Fusobacterium & 1 & 4 \\
\hline Gemella & 2 & 30 \\
\hline Granulicatella & 0 & 45 \\
\hline Haemophilus & 0 & 6 \\
\hline Lactobacillus $\dagger$ & 8 & ND \\
\hline Micrococcus $\dagger$ & 6 & ND \\
\hline Neisseria & 0 & 1 \\
\hline Pasteuriaceae incerta & 0 & 5 \\
\hline Pediococcus $†$ & 2 & ND \\
\hline Planomicrobium & 0 & 2 \\
\hline Porphyromonas & 0 & 6 \\
\hline Prevotella & 18 & 7 \\
\hline Propionibacterium & 3 & 1 \\
\hline Pseudomonas & 40 & 38 \\
\hline Ralstonia & 0 & 3 \\
\hline Rothia & 5 & 2 \\
\hline Staphylococcus & 20 & 1 \\
\hline Stenotrophomonas $\dagger$ & 1 & ND \\
\hline Streptococcus & 19 & 47 \\
\hline Variovorax $†$ & 2 & ND \\
\hline Veillonella & 33 & 27 \\
\hline Total & 211 & 251 \\
\hline
\end{tabular}

*Genera only included if they were detected by T-RFLP with a threshold score of $>0.2$ (see online supplement).

†Genera for which no T-RFLP reference spectra were generated and therefore could not be detected (ND) by T-RFLP analysis.
Culture-independent T-RFLP detection of microbial community composition and comparison with total viable counts from culture data

The composition of the bacterial community was also analysed in 64 sputum samples by T-RFLP (see online figure $1 \mathrm{~b}$ and online table 3 ). In general, all samples contained numerous terminal restriction fragments indicating that bacterial diversity within sputum samples was greater than detected by culture. In order to compare culture and T-RFLP results, T-RFLP patterns for each sample were compared with the patterns generated from $16 \mathrm{~S}$ rRNA gene clones (see table 2 in online supplement) representing 22 genera frequently detected in CF sputum. Based on this comparison, organisms belonging to the 22 genera were predicted to be present a total of 251 times among the 64 samples (figure 1 and table 2).

The predominant aerobic pathogens (Pseudomonas and Burkholderia) detected by culture were predicted by T-RFLP to occur in the corresponding patient samples (see figure $1 \mathrm{~b}$ in online supplement). Strict anaerobes were predicted by T-RFLP in $22 / 26$ admission samples, 20/25 completion of treatment samples and 9/ 13 stable samples with up to eight different anaerobic genera detected in a single sputum sample. Similar to the culture results, Veillonella and Prevotella were frequently detected. In addition, a number of anaerobic genera were detected exclusively by T-RFLP in multiple samples including Granulicatella (45/64), Porphyromonas (6/64) and Pasteuriaceae (5/64). Consistent with the culture-based results, molecular detection also showed that the composition of the bacterial community remained relatively stable in the majority of patients with similar T-RFLP patterns generated (see figure $1 \mathrm{~b}$ in online supplement) and similar numbers and genera of both aerobic and anaerobic species detected. Individual spectra taken at different time points from the same patient were comparable, demonstrating the reproducibility of the method (see figure 2 in online supplement).

The agreement between the presence or absence of bacteria from the T-RFLP spectra and TVCs from culture was relatively good for aerobic bacteria such as Paeruginosa $(\kappa=0.74)$ and $B$ cepacia complex $(\kappa=0.92)$ but poor for Streptococcus $(\kappa=0.05)$ and anaerobic genera such as Veillonella $(\kappa=0.14)$ and Prevotella $(\kappa=0.00)$.

Comparison of aerobe and anaerobe viable counts and effect of antibiotic treatment on bacterial abundance

Aerobic bacteria, cultured under the conditions used, were present in significantly greater numbers than anaerobes in samples at initiation of antibiotic treatment (median difference in TVC $4.9 \times 10^{7} \mathrm{cfu} / \mathrm{g}\left(95 \% \mathrm{CI} 1.8 \times 10^{7}\right.$ to $\left.14.5 \times 10^{7}\right) ; \mathrm{p}<0.001$, Wilcoxon signed rank test) and when patients were stable (median difference in TVC $3.8 \times 10^{7} \mathrm{cfu} / \mathrm{g}\left(95 \%\right.$ CI $1.1 \times 10^{7}$ to $\left.7.8 \times 10^{7}\right)$; $\mathrm{p}=0.01$ ) but less so at completion of antibiotic treatment (median difference in TVC $1.3 \times 10^{7} \mathrm{cfu} / \mathrm{g}\left(95 \% \mathrm{CI}-0.01 \times 10^{7}\right.$ to $\left.6.2 \times 10^{7}\right) ; p=0.07$; figure 2$)$. There was also a decrease in TVC 


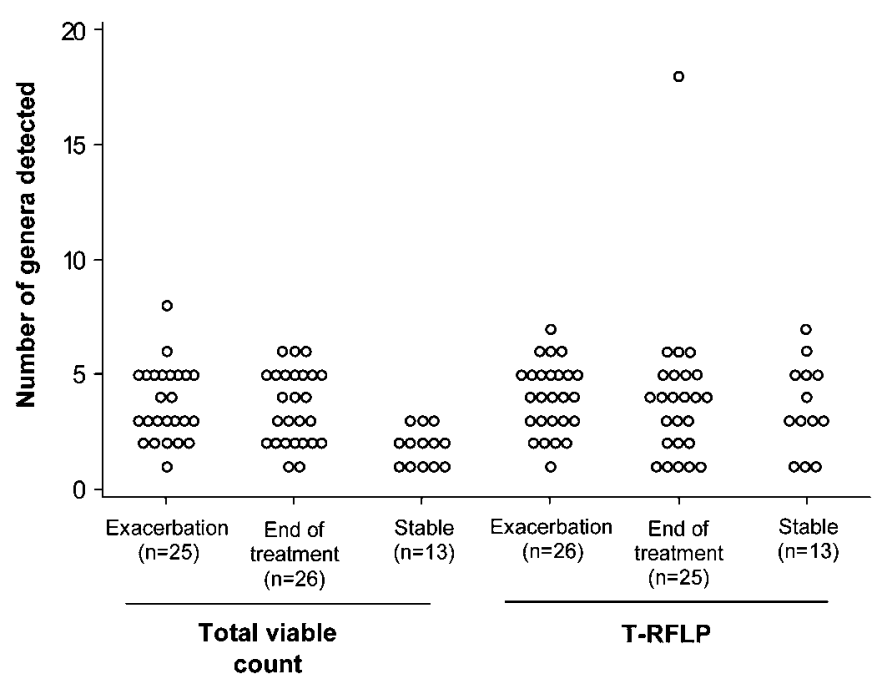

Figure 1 Number of genera detected by culture and terminal restriction fragment length polymorphism analysis (T-RFLP) in sputum samples collected from adult patients with cystic fibrosis at initiation (Exacerbation) and completion of intravenous antibiotic treatment (End of Treatment) for an exacerbation and when stable (Stable).

with antibiotic treatment for aerobic bacteria (median decrease in TVC $2.3 \times 10^{7} \mathrm{cfu} / \mathrm{g}\left(95 \%\right.$ CI $6.5 \times 10^{7}$ to $\left.12.8 \times 10^{7}\right) ; \mathrm{p}=0.005$, corresponding to a median percentage reduction of $85 \%$ ) and some evidence of a similar reduction for anaerobic bacteria (median decrease in TVC $3 \times 10^{6} \mathrm{cfu} / \mathrm{g}\left(95 \% \mathrm{CI} 0.3 \times 10^{6}, 7 \times 10^{6}\right)$; $\mathrm{p}=0.046$, corresponding to a median percentage reduction of $51 \%$; figure 2). Comparison of numbers (Friedman test) for the 13 patients in which three samples (stable, initiation and completion of antibiotic treatment) were collected revealed evidence of a difference in the aerobic $(p=0.016)$ but not the anaerobic bacteria $(p=0.15)$ between the three groups. To generate a more taxa-specific view of bacterial species in which abundance changed with antibiotic treatment, viable counts for individual species were compared at the different time points. $P$ aeruginosa TVC decreased from $6.7 \times 10^{7} \mathrm{cfu} / \mathrm{g}$ before antibiotic treatment to $2.5 \times 10^{7} \mathrm{cfu} / \mathrm{g}$ after antibiotic treatment for the 16 matched sample pairs in which Paeruginosa had been detected on admission for treatment (median decrease in TVC $2.0 \times 10^{7} \mathrm{cfu} / \mathrm{g}(95 \% \mathrm{CI}$ $0.5 \times 10^{7}$ to $\left.6.8 \times 10^{7}\right) ; \mathrm{p}=0.01$, corresponding to a median percentage reduction of $89 \%$; figure 3 ). No differences in TVC were apparent for any other species.

In addition to quantifying bacterial load with TVC, we also used qPCR, a low-cost highly sensitive and reproducible molecular technique targeting the conserved $16 \mathrm{~S}$ rRNA gene. ${ }^{16}$ While this method does not distinguish between bacterial species, there was evidence of a decrease in total bacterial concentration with antibiotic treatment (see figure 3 in online supplement) with the $\log _{10}$ qPCR value on average 0.53 (95\% CI 0.13 to 0.94 ) higher at initiation compared with end of treatment ( $p=0.01$, paired sample t test).

\section{DISCUSSION}

In this study we used both aerobic/anaerobic culture and molecular (T-RFLP) techniques to investigate microbial community diversity and abundance in sputum from patients with CF at the initiation and completion of antibiotic treatment for an acute exacerbation and when clinically stable. Multiple anaerobic species were detected by both methods, further confirming and expanding upon the findings of previous studies ${ }^{4-8} \quad 1011 \quad 18-20$ which have reported that a highly diverse polymicrobial

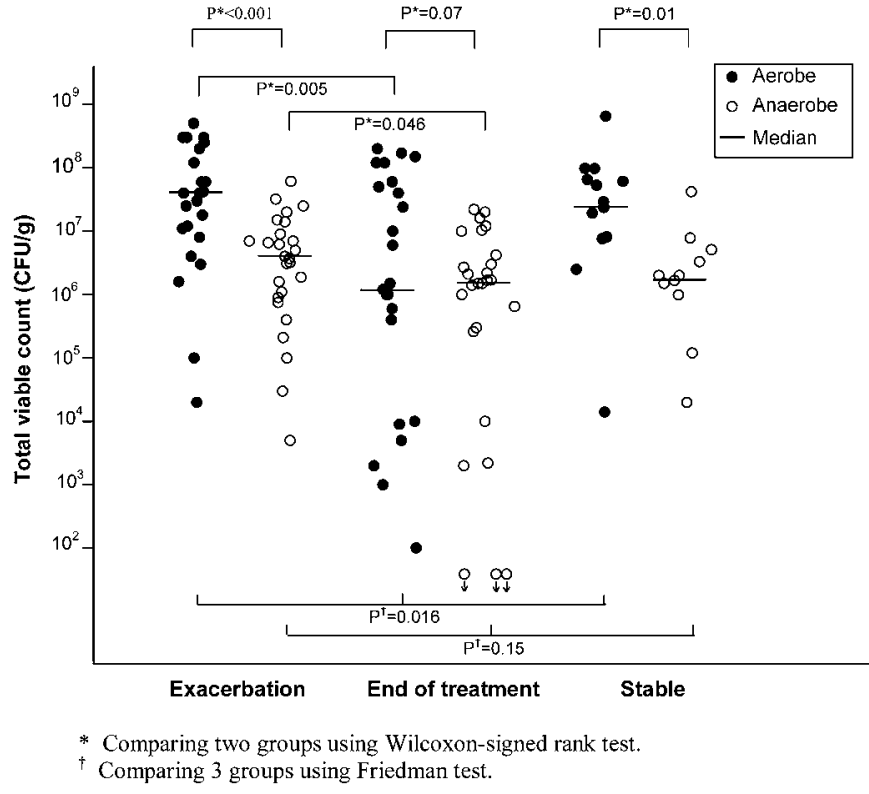

Figure 2 Total viable counts/g sputum of aerobic and anaerobic bacteria cultured from sputum samples collected from adult patients with cystic fibrosis at initiation (Exacerbation) and completion of IV antibiotic treatment (End of Treatment) for an exacerbation and when stable (Stable). CFU, colony-forming unit.

community exists within the CF lung. Furthermore, both methods demonstrate that the composition of the bacterial community remained relatively constant in the majority of patients before and after antibiotic treatment and when clinically stable, and that there was similarity in the composition between exacerbation and stable states. In a previous study of patients with stable CF we also demonstrated persistence of anaerobic species in multiple samples from the same patients. ${ }^{8}$ Unfortunately, owing to the small sample size, we were unable to determine if there was a correlation between the presence or absence of particular species and it is important that future studies with larger patient numbers address this issue. These results provide further evidence that patients with CF with established lung disease are chronically colonised by a range of bacterial species, including anaerobes, which persist despite antibiotic treatment.

Although the composition of the bacterial community remained relatively constant, our quantitative culture results suggest that changes in bacterial abundance can be detected following antibiotic treatment, with this effect being more pronounced for aerobes than anaerobes. This finding was confirmed by qPCR which showed a decrease in bacterial $16 \mathrm{~S}$

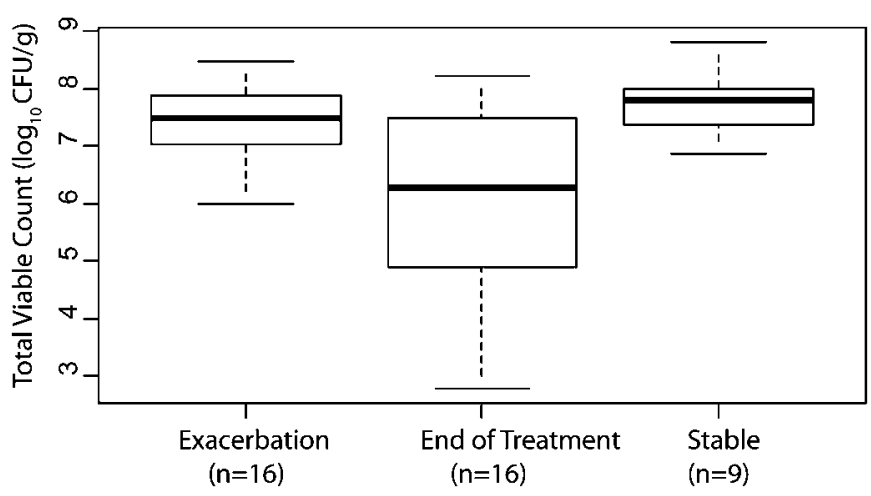

Figure 3 Comparison of total viable counts/g sputum for Pseudomonas aeruginosa. CFU, colony-forming unit. 
rRNA gene copies/g sputum following antibiotic treatment. Furthermore, our results also suggest that $P$ aeruginosa was more susceptible to current antibiotic therapy than other members of the microbial community as it was only the genus in which there was a significant decrease in abundance with antibiotic treatment. This may be because antibiotic treatment in the majority of patients was targeted against $P$ aeruginosa and that the antibiotics primarily used (tobramycin and ceftazidime) have poor in vitro activity against anaerobic bacteria. ${ }^{10} 21$ Our findings are consistent with previous reports which showed that a decrease in bacterial load in sputum following antibiotic treatment is associated with clinical improvement. ${ }^{22-25}$ Exacerbations were treated with a wide range of antibiotic regimens, so we were uable to determine if there was a relationship between the use of specific antibiotics such as meropenemwhich has excellent in vitro activity against anaerobes cultured from CF sputum ${ }^{8}{ }^{10}$-and the change in anaerobic bacterial load following treatment.

There was relatively good agreement between T-RFLP and culture detection methods for the identification of the more abundant aerobic pathogens, $P$ aeruginos $a$ and $B$ cepacia complex. This finding confirms the results of previous studies which also reported excellent congruence between aerobic culture and bacterial rRNA gene detection methods. ${ }^{71}$ However, in contrast to the findings for aerobic bacteria, there was poor agreement between $\mathrm{T}$ RFLP and TVC culture data for anaerobes which were present in greater diversity and lower abundance. These findings suggest that these very different methods are similarly effective at detecting abundant organisms but both are required to capture the full spectrum of less abundant and potentially difficult to culture anaerobes. As our identification prediction involves a number of bacterial taxa that are poorly characterised and under-represented in $16 \mathrm{~S}$ rRNA gene sequence databases, we restricted our identification to the genus level. Future identification of anaerobic bacteria to species level may be important when assessing the potential pathogenicity of any anaerobes present. This further emphasises both the importance of culture in the detection of anaerobic bacteria and that the two methods are complementary in the data they provide. T-RFLP assignments that had no corresponding culture assignment may have been due to the presence in the samples of bacteria which were not cultured by the methods employed. Furthermore, bacterial genera predicted by T-RFLP were limited to those organisms for which reference patterns were available. Inclusion of additional reference spectra would therefore increase the range of bacterial genera identified by T-RFLP. However, more expensive methods such as deep sequencing ${ }^{26}$ and microarrays (eg, 16S rRNA PhyloChip ${ }^{27}$ ) may allow more comprehensive characterisation of CF lung microbial communities.

In the present study, streptococci from the Streptococcus milleri group (SMG) and the $S$ viridans group were identified in 19 samples by culture, with streptococci detected by T-RFLP in 47/ 64 samples. This was not unexpected given that our study focused on anaerobic bacteria and that we did not use selective media or incubation conditions to optimise the culture detection of streptococci. Previous studies have similarly shown that streptococci from the SMG may not be detected by routine CF microbiology protocols. ${ }^{28}$ A case report study ${ }^{29}$ and a more recent longitudinal study ${ }^{28}$ have both shown a beneficial clinical response to treatment of the predominant SMG organism during exacerbations, suggesting that streptococci may also contribute to a polymicrobial community of bacteria that are CF respiratory pathogens. Culture protocols in subsequent studies should therefore be amended to optimise the detection and quantitation of these organisms.
The potential pathogenic role of bacteria such as anaerobes and streptococci in CF pulmonary infection remains unclear. However, some of these anaerobic bacteria have been shown to be pathogenic in other lung diseases ${ }^{30-32}$ and preliminary studies by our group have shown that they are also present in the lungs of patients with non-CF bronchiectasis. ${ }^{33}$ Ultimately, the clinical relevance of anaerobic bacteria in the CF lung can only be addressed by clinical trials in which patients receive antibiotics targeted against aerobic bacteria only versus antibiotics targeted against both aerobes and anaerobes. Subsequent determination of the reduction in bacterial load combined with analysis of pulmonary function and markers of inflammation could establish whether anti-anaerobe treatment resulted in improved clinical outcome for patients. However, some antibiotics with putative antimicrobial activity against anaerobic bacteria are not effective in vitro against anaerobes isolated from CF sputum. ${ }^{810}$ Antibiotic regimens used in such trials would therefore need to take account of the distinctive sensitivity patterns of anaerobes present in the CF lung.

In conclusion, our results show that we can detect changes in bacterial abundance within the microbial community following antibiotic treatment but that these changes are only large enough to be detected individually for $P$ aeruginosa. They also show that aerobic/anaerobic culture and molecular detection methods are both required for comprehensive characterisation of the microbial community in the CF lung. Molecular methods are sensitive and accurate and provide a more complete view of the bacterial communities present and may also be useful to direct improved species-specific culture methods. However, they do not remove the need for culture detection which is more quantitative and provides the opportunity to perform antibiotic sensitivity testing which can subsequently be used to target antibiotic treatment. Further larger studies of polymicrobial CF lung infection, including alteration of current antibiotic treatment in CF to treat these newly identified bacteria, are urgently required to elucidate more fully their clinical significance and improve treatment strategies and outcome for patients with CF.

Acknowledgements The authors acknowledge the technical assistance of $\mathrm{Mr}$ Gerry McGrillen (School of Pharmacy, Queen's University Belfast). We also wish to acknowledge Professor John Moore (Belfast Health and Social Care Trust) for helpful discussions in respect of this study.

Funding Supported by a UK Cystic Fibrosis Trust grant (PJ533) and a grant from the US National Institutes of Health (HL084934). MMT was supported by a Health and Social Care Research and Development, Public Health Agency, Northern Ireland-funded UK National Institute for Health Research Career Scientist Award.

\section{Competing interests None.}

Ethics approval This study was conducted with the approval of the Office for Research Ethics Northern Ireland.

Provenance and peer review Not commissioned; externally peer reviewed.

\section{REFERENCES}

1. Dodge JA, Lewis PA, Stanton M, et al. Cystic fibrosis mortality and survival in the UK: 1947-2003. Eur Respir J 2007;29:522-6.

2. Ratjen F. Changes in strategies for optimal antibacterial therapy in cystic fibrosis. Int J Antimicrob Agents 2001;17:93-6.

3. Worlitzsch D, Tarran R, Ulrich $\mathrm{M}$, et al. Effects of reduced mucus oxygen concentration in airway Pseudomonas infections of cystic fibrosis patients. J Clin Invest 2002:109:317-25.

4. Rogers GB, Carroll MP, Serisier DJ, et al. Characterization of bacterial community diversity in cystic fibrosis lung infections by use of 16 s ribosomal DNA terminal restriction fragment length polymorphism profiling. J Clin Microbiol 2004; 42:5176-83.

5. Rogers GB, Carroll MP, Serisier DJ, et al. Bacterial activity in cystic fibrosis lung infections. Respir Res 2005:6:49.

6. Rogers GB, Carroll MP, Serisier DJ, et al. Use of $16 \mathrm{~S}$ rRNA gene profiling by terminal restriction fragment length polymorphism analysis to compare bacterial communities in sputum and mouthwash samples from patients with cystic fibrosis. J Clin Microbiol 2006;44:2601-4. 
7. Harris JK, De Groote MA, Sagel SD, et al. Molecular identification of bacteria in bronchoalveolar lavage fluid from children with cystic fibrosis. Proc Natl Acad Sci U S A 2007:104:20529-33

8. Tunney MM, Field TR, Moriarty TF, et al. Detection of anaerobic bacteria in high numbers in sputum from patients with cystic fibrosis. Am J Respir Crit Care Med 2008;177:995-1001.

9. Tunney MM, Klem E, Gilpin DF, et al. Detection of anaerobic bacteria in bronchoalveolar lavage fluid from paediatric CF patients. J Cyst Fibros 2009;8 (Suppl 2):S45

10. Worlitzsch D, Rintelen C, Bohm K, et al. Antibiotic-resistant obligate anaerobes during exacerbations of cystic fibrosis patients. Clin Microbiol Infect 2009:15:454-60.

11. Bittar F, Richet $\mathrm{H}$, Dubus JC, et al. Molecular detection of multiple emerging pathogens in sputa from cystic fibrosis patients. PLOS ONE 2008;3:e2908.

12. Rogers GB, Skelton S, Serisier DJ, et al. Determining CF lung microbiology: a comparison of spontaneous and serially induced sputum samples using T-RFLP profiling. J Clin Microbiol 2010:48:78-86.

13. Amann RI, Ludwig W, Schleifer KH. Phylogenetic identification and in situ detection of individual microbial cells without cultivation. Microbiol Rev 1995;59:143-69.

14. Fuchs HJ, Borowitz DS, Christiansen DH, et al. Effect of aerosolized recombinant human DNase on exacerbations of respiratory symptoms and on pulmonary function in patients with cystic fibrosis. The Pulmozyme Study Group. N Engl J Med 1994;331:637-42.

15. Lipuma JJ, Dulaney BJ, McMenamin JD, et al. Development of rRNA-based PCR assays for identification of Burkholderia cepacia complex isolates recovered from cystic fibrosis patients. J Clin Microbiol 1999;37:3167-70.

16. Nadkarni MA, Martin FE, Jacques NA, et al. Determination of bacterial load by realtime PCR using a broad-range (universal) probe and primers set. Microbiology 2002;148(Pt 1):257-66.

17. Rogers GB, Hart CA, Mason JR, et al. Bacterial diversity in cases of lung infection in cystic fibrosis patients: $16 \mathrm{~S}$ ribosomal DNA (rDNA) length heterogeneity PCR and 16S rDNA terminal restriction fragment length polymorphism profiling. J Clin Microbiol 2003;41:3548-58.

18. Jewes LA, Spencer RC. The incidence of anaerobes in the sputum of patients with cystic fibrosis. J Med Microbiol 1990;31:271-4.

19. Brook I, Fink R. Transtracheal aspiration in pulmonary infection in children with cystic fibrosis. Eur J Respir Dis 1983;64:51-7.
20. Thomassen MJ, Klinger JD, Badger SJ, et al. Cultures of thoracotomy specimens confirm usefulness of sputum cultures in cystic fibrosis. J Pediatr 1984;104:352-6.

21. Aldridge KE. The occurrence, virulence, and antimicrobial resistance of anaerobes in polymicrobial infections. Am J Surg 1995:169(5A Suppl):2S-7S

22. Gibson RL, Emerson J, McNamara S, et al. Significant microbiological effect of inhaled tobramycin in young children with cystic fibrosis. Am J Respir Crit Care Med 2003:167:841-9.

23. Smith AL, Doershuk C, Goldmann D, et al. Comparison of a beta-lactam alone versus beta-lactam and an aminoglycoside for pulmonary exacerbation in cystic fibrosis. J Pediatr 1999;134:413-21.

24. Regelmann WE, Elliott GR, Warwick WJ, et al. Reduction of sputum Pseudomonas aeruginosa density by antibiotics improves lung function in cystic fibrosis more than do bronchodilators and chest physiotherapy alone. Am Rev Respir Dis 1990;141 (4 Pt 1):914-21.

25. Blumer JL, Saiman L, Konstan MW, et al. The efficacy and safety of meropenem and tobramycin vs ceftazidime and tobramycin in the treatment of acute pulmonary exacerbations in patients with cystic fibrosis. Chest 2005:128:2336-46.

26. Armougom F, Bittar F, Stremler N, et al. Microbial diversity in the sputum of a cystic fibrosis patient studied with $16 \mathrm{~S}$ rDNA pyrosequencing. Eur J Clin Microbiol Infect Dis 2009;28:1151-4.

27. Huang YJ, Kim E, Cox MJ, et al. A persistent and diverse airway microbiota presen during chronic obstructive pulmonary disease exacerbations. OMICS;14:9-59.

28. Sibley CD, Parkins MD, Rabin HR, et al. A polymicrobial perspective of pulmonary infections exposes an enigmatic pathogen in cystic fibrosis patients. Proc Natl Acad Sci U S A 2008;105:15070-5

29. Cade A, Denton M, Brownlee KG, et al. Acute bronchopulmonary infection due to Streptococcus milleri in a child with cystic fibrosis. Arch Dis Child 1999;80:278-9

30. Dore $\mathbf{P}$, Robert $\mathrm{R}$, Grollier $\mathrm{G}$, et al. Incidence of anaerobes in ventilator-associated pneumonia with use of a protected specimen brush. Am J Respir Crit Care Med 1996;153(4 Pt 1):1292-8.

31. Hammond JM, Potgieter PD, Hanslo D, et al. The etiology and antimicrobial susceptibility patterns of microorganisms in acute community-acquired lung abscess Chest 1995:108:937-41.

32. Brook I, Frazier EH. Aerobic and anaerobic microbiology of empyema. A retrospective review in two military hospitals. Chest 1993;103:1502-7.

33. Drain M, Wei L, Einarsson G, et al. Detection of anaerobic bacteria in stable noncystic fibrosis bronchiectasis patients. Am J Respir Crit Care Med 2010;181:A3177.

\title{
Journal club
}

\section{CFTR-knockout neonatal ferrets model may be useful in the understanding of CF pathogenesis and developing therapies}

There is no adequate animal model of cystic fibrosis (CF) airways disease. This study investigated using CFTR-knockout neonatal ferrets as they show similarities to newborn humans with CF. These similarities include meconium ileus (MI), pancreatic disease, liver disease, severely impaired nutritional status, absent or degenerate vas deferens at birth, defective airways and a predisposition of lung infection. There is a higher prevalence of MI and intestinal complications in CFTR-knockout kits than in humans with CF. This limitation is overcome by using transgenic CFTR-knockout kits expressing CFTR in the intestine. Oral administration of ursodeoxycholic acid normalised liver function and a proton pump inhibitor improved the nutrition status and survival in the CF ferrets. Despite improved nutrition, CFTR-knockout neonatal ferrets had increased bacterial counts in the airways that subsided after the first week.

The findings of CF phenotype in various organs in CFTR-knockout neonatal ferrets and their similarities to human CF may be useful in the understanding of CF pathogenesis and the future development of organ-specific therapies.

- Sun X, Sui H, Fisher JT, et al. Disease phenotype of a ferret CFTR-knockout model of cystic fibrosis. J Clin Invest 2010;120:3149-60.

\author{
Albert YH Lim \\ North West Midlands Cystic Fibrosis Centre, University Hospital of North Staffordshire, Stoke on trent, UK \\ Correspondence to Dr Albert YH Lim, North West Midlands Cystic Fibrosis Centre, University Hospital of North \\ Staffordshire, UK; albert.lim@uhns.nhs.uk
}

Published Online First 22 October 2011

Thorax 2011;66:584. doi:10.1136/thx.2010.151910 